\title{
Understanding Behaviors and Needs for Home Videos
}

\author{
Marco Campanella \\ Philips Research Europe \\ High Tech Campus 34, Postbox WB41 \\ 5656 AE Eindhoven - The Netherlands \\ +31402747899 \\ marco.campanella@philips.com
}

\author{
Jettie Hoonhout \\ Philips Research Europe \\ High Tech Campus 34, Postbox WB51 \\ 5656 AE Eindhoven - The Netherlands \\ +31402747512 \\ jettie.hoonhout@philips.com
}

\begin{abstract}
Nowadays, people capture, edit and share more and more home videos. Devices with video capturing capability and services for video sharing and editing are becoming increasingly popular. This study was conducted to chart in more detail what users currently do with their home videos, and what their desires, problems and needs are. The results of an Internet-based survey learned that home videos have a social function. They are used to keep memories of group experiences and watched mainly with groups of interested acquaintances. Furthermore, home videos are shared and edited quite frequently. The insights we report here can serve as starting points for designing better and more helpful systems for managing home videos.
\end{abstract}

\section{Categories and Subject Descriptors}

H.5.2 [Information Interfaces and Presentation (e.g., HCI)]: User Interfaces - User-centered design.

\section{General Terms}

Measurement, Human Factors.

\section{Keywords}

Home video, user needs, usage habits, internet survey.

\section{INTRODUCTION}

Nowadays, more and more often home videos are created, edited and shared. Sales of digital still cameras and mobile phones that enable video capturing are increasing. To help users manage and edit their home videos, several PC programs are available on the market, such as Adobe Premiere, Windows Movie Maker. In the last years new tools and web-based services have appeared with new ways of handling home videos. The program Muvee, for example, enables fully automatic home video editing; on the Internet people can share videos via YouTube, create video-blogs, and edit videos with services like Jumpcut.

To help users with their home videos, a number of systems for fully-automatic and semi-automatic video editing has been proposed in the fields of multimedia content analysis and management. However, the amount of research aimed at clarifying users' habits and needs related to personal videos is

(C) The Author 2008.

Published by the British Computer Society very limited. With this study we aim to chart a detailed picture of how and why people capture, watch, edit and share home videos, and of their problems and needs. This is important information when designing tools for home videos that address the users' needs.

This paper is organized as follows. In Section 2 the relevant literature is presented and discussed. In Section 3 the goals of our research are stated and the method of investigation is explained. In Section 4 the results are presented, and in Section 5 conclusions and future developments are discussed.

\section{RELATED LITERATURE}

Many solutions have been proposed in literature to help users capture, handle and edit their home videos. A number of studies have been dedicated to provide users with systems for automatic home video editing. In these systems, content analysis algorithms are exploited to automatically analyze and structure raw video material. Successively, clips are selected and combined in an edited version of the video, which is finally enriched with music and video effects and presented to the user. The algorithms in [3], [5] and [7] automatically summarize and edit a video, using different criteria. The summarization is performed by selecting clips with high video quality or with particular features like faces, camera motion, and object motion.

Other studies have focused on systems for semi-automatic home video editing. The assumption underpinning semiautomatic editing system is that users want to edit videos in a fast and easy way, while having at the same time some control on the editing process. Semi-automatic systems try to automate the most time-consuming and difficult operations in video editing, like frame-precise cutting or removal of low-quality video clips. Normally these systems provide structure to the raw video material, dividing it into meaningful portions. The user is then given the possibility to use the system to perform highlevel editing operations, like choosing portions of video to compose an edited version. Different interfaces for selecting pre-segmented portions of video to be included in the edited versions have been developed, and evaluated in use tests; see for example [1] and [2].

Many of these automatic and semi-automatic systems are also evaluated in use tests. However these tests are designed to assess the usability of the systems, without specifically addressing the user needs related to home videos. In these studies, only assumptions about the user needs are presented. These assumptions come back with little variations in all the cited papers. According to [5] for example, home videos easily sum up to many hours, most of them are never touched or watched again because the unedited video footage has low quality and is not appealing. Video editing could help, but is too time-consuming and therefore rarely done. Users would like different versions of their videos for different people. 
At the time of writing we are aware of only one study ([4]) that tries to verify which of the aforementioned assumptions are true and which not, focusing on how people use their home videos and what are their needs and problems. The authors of [4] have conducted interviews with 18 subjects from 12 families in their homes and a focus-group with 7 teenagers. The aim of their study was to collect information about the whole lifecycle of home videos: choosing the device, capturing, editing, consuming. The authors conclude that two ways of working with home videos can be distinguished: the lightweight and the heavyweight videowork. In the lightweight videowork, users exploit mobile phones and digital still cameras to spontaneously shoot short clips, without pre-planning, to capture on-the-fly interesting happenings. These short clips are mostly not edited, are shown in the capture devices or shared via Internet. In the heavyweight videowork camcorders and digital still cameras are used to shoot long clips of important events, in a deliberate and planned way. These longer videos are edited more often, in order to demonstrate the user's creativity and to obtain a tangible result like a DVD. Although interesting, unfortunately the study is based on only a small number of respondents.

In the literature we just reviewed, we observe a lack of concern with respect to the role home videos have in the lives of users and a lack of data on their real needs. This study is meant to address these points.

\section{SURVEY}

Our main objective was to study why and how people capture, watch, share and edit home videos. Furthermore, we were interested in eliciting the wishes and needs of users of home videos. Answering these questions can be very useful for designing technology that helps users more effectively and addresses their problems. To address the aforementioned research questions, we decided to implement an Internet-based questionnaire, to facilitate easy distribution of the survey, and support data collection in a convenient way. The questionnaire, an English and an Italian version, has been distributed via bulletin boards and mailing lists at Universities and the Eindhoven Research Campus. Anyone who had at least a basic experience in shooting home videos with any device was invited to take part in our survey.

\section{RESULTS}

\subsection{Respondents' profile}

The questionnaire was answered by 181 people, $67 \%$ of which are living in the Netherlands, the remaining $33 \%$ in Italy. $69 \%$ of the respondents were male. Given the distribution channels we employed, it is not surprising that the majority of the respondents is relatively young, and that many of the respondents indicated to have a background related to technical disciplines. $73 \%$ of the respondents are younger than 36 . However, the number of respondents in our sample older than 36 (49 respondents) still enabled us to obtain relevant insights in how home video use might change when people's lifestyle is changing when getting older and e.g. starting families.

We asked the participants the level of experience they have with PCs. We decided to divide the sample into two groups of participants: users of basic PC applications like Internet, email and word processing (54 respondents), and users of more advanced PC applications, or IT professionals (107 respondents). The fact that our sample included more respondents with a high level of PC experience will probably be due to the technical background of many of our participants, among which the questionnaire was spread. In our sample, the respondents with more PC experience turned out to work with home videos more frequently. We have not been able to verify if our sample differs in this respect from the general population of home video users.

\subsection{Relevant subgroups of participants}

In order to determine whether particular participant characteristics result in different response patterns, we compared the questionnaire results of different user groups:

- $\quad$ Respondents older than 36 (49 people).

- Respondents younger than 36 (132 people).

- Respondents with low PC expertise (54 people).

- $\quad$ Respondents with high PC expertise (107 people).

- $\quad$ Respondents that capture mostly short $\left(<15^{\prime}\right)$ videos $(89$ people).

- Respondents that capture mostly long $\left(>15^{\prime}\right)$ videos $(85$ people).

Given the characteristics of the data set a Chi-square test for independence was seen as most appropriate [6] to analyze the answer patterns of the subgroups as mentioned, with $\alpha=0.05$. For clarity we report the Chi-square data only for the main result, however all results concerning the user groups have been validated by test of independence.

\subsection{Capturing home videos}

Our respondents capture home videos with diverse frequencies. $52 \%$ of respondents capture home videos five times a year or less, the remaining $48 \%$ capture home videos at least six times a year (7\% at least once a week). People capture mostly social events: holidays $(65 \%)$, parties or events with friends $(58 \%)$, family life $(46 \%)$, artistic events such as concerts $(19 \%)$, sport events $(17 \%)$, weddings $(13 \%)$ and nature scenes $(4 \%)$. Age seems to influence the frequency of capturing family life and events with friends. People older than 36 shoot more frequently family life $\left(69 \%, \chi^{2}=14.26\right.$, df. $\left.=1\right)$, while participants younger than 36 capture more often events with friends $(68 \%$, $\chi^{2}=22.94$, df. $\left.=1\right)$ - basically reflecting a shift in lifestyle from being single or a couple without children to one in which one has started a family with children.

We also asked which devices people use to capture videos. Digital still cameras $(59 \%$ of respondents), and digital camcorders $(57 \%)$ are most popular, but also mobile phones are used $(28 \%)$. The camcorder is used more by people older than 36 than by participants younger than 36 . The digital still camera and the mobile phone are more appreciated by younger respondents.

The duration of a home video depends on the type of event. The time duration of videos concerning weddings and holidays distributes uniformly from $5^{\prime}$ to more than $90^{\prime}$. On the other hand, people capture other life with friends and with the family in relatively short videos, no longer than 45 minutes. A reason might be that people tend to plan the capture of long events like holidays and weddings, while the capture of events with friends and family happens more spontaneously and "on the fly".

People indicate as main reasons for using home videos to keep memories of someone's life $(86 \%)$ and to share experiences with acquaintances $(51 \%)$. Also, people agree that they would like to watch their home videos on TV. Despite the widely accepted assumption that home videos are not watched after capturing because of their low quality, it appears that the video quality of personal audiovisual documents does not have a big influence on the pleasantness of the videos. Only $29 \%$ of 
respondents find home videos not appealing because of the low quality. This figure does not change significantly for users of different capturing devices. A couple of respondents even commented that they consider low video quality of home videos as a feature that makes home videos unusual and interesting.

\subsection{Watching home videos}

Once a home video is captured, it is watched mostly around 2 to 5 times. Participants were offered the option to specify whether the watching frequency is dependent on aspects of the videos and aspects of the users' social life. $32 \%$ of respondents spontaneously described what influences the frequency of watching home videos. Among these respondents, $45 \%$ pointed out that they watch particular events more often than others; the type of event of a home video is of crucial importance when deciding whether to view the video. Another 23\% said that some videos are watched more often because they are requested by others: children, friends, family members, acquaintances. Other factors less important in influencing whether or not a home video will be watched more than once, are the level of interest (is the video amusing, funny, or boring), the accessibility (whether it is easily accessible on a DVD or in a video file), the duration, the quality, the time users have available.

Watching home videos appears to be done mostly in groups. Only $21 \%$ of respondents watch home videos mostly alone; the rest watches home videos mostly with family or friends. Participants older than 36 watch home videos more frequently with the family $\left(\chi^{2}=23.29\right.$, df. $\left.=1\right)$. On the other hand, people younger than 36 watch home videos more frequently with friends $\left(\chi^{2}=13.47\right.$, df. $\left.=1\right)$. As seen before, respondents explicitly remarked that frequent reasons for watching home videos are children, family or friends. Thus, it appears that people see the experience of watching home videos as a social/communicative moment, to be shared with the group of dear ones, family members or friends.

The device used for watching depends on the duration of the video. Long and short videos are watched equally often on a PC, but the situation is very different for the TV. While $85 \%$ of owners of long videos use the TV as viewing device, only $26 \%$ of users with short videos use the TV, although it appears that $43 \%$ of owners of short videos would like to watch these on $\mathrm{TV}$. We will come back on this later on.

\subsection{Sharing home videos}

Nowadays many people share their videos with others by using Internet, DVDs, USB-based means, etc. People appear to share videos quite frequently: $66 \%$ of our respondents "give away" copies of at least some of their personal videos. Respondents share home videos mainly with family members and friends, secondly with everyone who might be interested, by making the videos public. In line with what happens for capturing and watching, people younger than 36 share videos more frequently with friends $\left(\chi^{2}=16.75\right.$, df. $\left.=1\right)$. People older than 36 do not share their videos significantly more often with their family $\left(\chi^{2}\right.$ $=2.05$, df. $=1$ ). We also note that only $3 \%$ of users with low PC expertise make their home videos public, compared to $33 \%$ of users with high PC expertise $\left(\chi^{2}=11.42\right.$, df. $\left.=1\right)$.

We have asked the respondents who never share home videos what the most important reasons are for keeping their personal movies private. People complain that sharing videos takes too much time $(38 \%)$, big video files are too difficult to send $(35 \%)$, personal home videos are too private to share $(33 \%)$. $35 \%$ of non-sharers said that they never considered the possibility of sharing videos. It is interesting to note that reasons for not sharing videos are often technical difficulties, rather than e.g. privacy concerns.

\subsection{Editing home videos}

Our respondents edit their videos quite often. $64 \%$ of the respondents edit at least some of their videos. The frequency of editing home videos is dependent on the acquaintance users have with computers $\left(\chi^{2}=16.25\right.$, df. $\left.=3\right) .76 \%$ of people with high computer expertise edit some of their videos, against $46 \%$ of users with low computer expertise. Although our sample of participants may be biased towards people with high PC experience, we observe that even among users with low PC skills the editing frequency is surprisingly high, in contrast with what is commonly assumed in literature. The editing frequency is also correlated with the duration of home videos $\left(\chi^{2}=17.83\right.$, df. $=3$ ); $79 \%$ of owners of long videos do some editing, against $49 \%$ of creators of short videos. The editing frequency is not significantly correlated with the participants' age. We asked people who never or rarely edit their videos whether there is a reason for their low editing activity. Only $19 \%$ of them say that home videos do not need editing. The others complain about the amount of time required for video editing or about its difficulty.

Our survey contained a number of questions that only had to be answered by the $64 \%$ of respondents with at least some experience in video editing. These questions concerned the motivations for doing video editing, the time needed, the tools used and their main limitations. $89 \%$ of video editors said that the main reason for editing is to remove uninteresting parts: the raw video is normally too long. $67 \%$ of video editors enrich their videos by adding music and video effects, to increase the attractiveness of the video. Other motivations are to add a personal touch to the video (41\%), to have a nice video memory to keep (24\%), to have different versions of a video to give to different people (15\%). Owners of short videos plan much less to keep videos as a memory ( $14 \%$ against $31 \%$ of long videos).

Many respondents stated that video editing jobs take much time: $40 \%$ of video editors need 10 or more times longer for editing than the total duration of the raw material. We did not find any dependencies between time spent on editing and editing reasons. We asked also about the editing tools that are used most often. $66 \%$ of respondents use semi-professional tools like Adobe Premiere or Pinnacle Studio. 40\% of respondents use tools like Windows Movie Maker, Apple iMovie, Ulead Video Studio and similar programs, generally seen as a bit more user-friendly. $10 \%$ of respondents use editing tools embedded on hard disk recorders or DVD recorders. Table 1 shows the issues around the editing tools most often experienced by the users.

Table 1. Limits of video editing tools.

\begin{tabular}{|l|c|}
\hline Video editing tools are too time-consuming. & $56 \%$ \\
\hline I'm fully satisfied with my video editing tool. & $25 \%$ \\
\hline Video editing tools are too complex and technical & $23 \%$ \\
\hline $\begin{array}{l}\text { I cannot navigate and search well enough in my } \\
\text { videos }\end{array}$ & $18 \%$ \\
\hline $\begin{array}{l}\text { Video editing tools don't allow me to express fully } \\
\text { my creativity }\end{array}$ & $18 \%$ \\
\hline
\end{tabular}

Importantly, more than half of video editors observe that editing tools are too time-consuming, by far the main complaint. $44 \%$ of video editors with low PC expertise find editing programs too complex and too technical, against $20 \%$ of users with high PC skills. 


\subsection{Users' needs and wishes}

In our survey, participants were invited to rate their level of interest for a number of possible needs and wishes related to home video editing. Respondents indicated a clear interest for "combining own videos with own pictures, friends' content or content from the Internet", "changing and manipulating a video while watching it", and "editing videos on a TV". Owners of long videos expressed significantly more interest for editing their audiovisual memories on a TV. The respondents of our questionnaire were also invited to freely write down their needs and wishes related to home video editing. Some ideas concerned the possibility of involving others in the editing process. Other participants expressed the desire for more userfriendliness, of better ways of organizing and annotating videos and of compiling one movie from many small clips.

\subsection{Discussion}

Generally, it appears that home videos have a strong social role. People capture mostly social events, or experiences spent with a group of people that are important, friends for younger users and family for older users. Home videos are captured mainly for keeping memories of group experiences and sharing them, moreover home videos are watched principally with the group of people close to the user. Home videos are watched more or less often according to the type of event and frequently on demand of friends or family members; whether a video is watched or not is not primarily influenced by its quality. Home videos are shared very frequently, and apparently they would be shared even more if the technology was more efficient and easier to use. The fact that many users are interested in combining their audiovisual memories with friends' content or public content might be due to the social role of home videos.

Users younger than 36 present different habits than users older than 36. Younger users focus their capturing, watching and sharing activities more on the group of friends. They use more often digital still cameras and mobile phones for capturing videos, the PC for watching videos and USB-based means for sharing. Older users adopt much more often camcorders, their capturing and watching activity is more centered on the family. They use more often the TV for watching videos and DVDs for sharing. Our participants have frequently remarked that videos with family life (for older users) and experiences with friends (for younger users) are watched more often than other events such as holidays. Videos involving people close to the user are watched repeatedly to show to interested persons, or at the request of interested persons. More individual experiences tend to be watched only once or twice.

Video editing is done surprisingly often by our participants, although more than half of them remark that it takes too much time. The complexity of editing and the presence of difficult technical details are also perceived as problems. Perhaps video editing technology should take the social function of home videos more into account.

Users who shoot long videos and owners of short videos show different behaviors in dealing with their video memories. While long videos are usually watched on TV, only $26 \%$ of users of short videos watch them on TV. However, $43 \%$ of users of short videos would like to watch these on TV and $47 \%$ are interested in editing videos on TV. Moreover, short videos are edited much less often than long videos. From our study it appears also that, despite the growth of YouTube or of web services for videos, short videos are not shared significantly more often than long ones $\left(\chi^{2}=4.70\right.$, df. $\left.=3\right)$. Users of short videos are also less motivated to keep the videos $\left(\chi^{2}=4.52\right.$, df.
$=1$ ). It is possible that short videos are not watched on TV, edited and kept because they are seen as temporary snapshots of specific moments. However, it is also possible that people do not edit and keep short videos because technology tailored to effectively manage collections of short clips is lacking. It would be interesting to explore better systems for viewing videos taken with digital still cameras or mobile phones on TV, maybe organized in a DVD to be browsed by time or by scene, overcoming the problems due to the low resolution.

Finally, the complexity of software applications is a barrier in handling home videos. Users with high PC expertise are almost two times more likely to edit videos than users with low PC skills. Moreover, making home videos public via Internet-based means is done only by users with high PC expertise, while sharing with DVDs or USB-based means is done independently on the PC expertise.

\section{CONCLUSIONS}

We have run an Internet-based questionnaire to investigate the users' habits and usage patters related to home videos. The number of respondents has allowed us to obtain a relatively robust picture of different usage habits and needs related to home videos. This study indicated that home videos have principally a social/communicative function; people use them as memories for the group of family or friends. As concluded by [4], long videos and short videos appear to be handled in different ways. The assumption that home video editing is seen as too time-consuming and difficult is also confirmed. Home videos are shared and edited quite frequently. We plan to deepen our results by successive interviews and usability tests on user-friendly video editing systems for TV. We will also explore how to design an editing system taking into account the social role of personal videos, how to better exploit the clips of short duration taken with still cameras or mobile phones.

\section{ACKNOWLEDGEMENTS}

This work is performed as part of a Marie Curie Early Stage Training grant (MEST-CT-2004-8201).

\section{REFERENCES}

[1] Casares, J., et al., Simplifying video editing using metadata. In Proc. of the $4^{\text {th }}$ conference on Designing Interavtive Systems: processes, practices, methods and techniques, 2002. 157-166.

[2] Girgensohn, A., et al., Home Video Editing Made Easy Balancing Automation and User Control. In Proc. of Int. Conf. on Human Computer Interaction (INTERACT '01), Japan. 464-471

[3] Hua, X.-S., et al., Optimization-Based Automated Home Video Editing system. IEEE Transactions on Circuits and Systems for Video Technology 2004, 572-583.

[4] Kirk, D., et al., Understanding Videowork. In Proc. of the SIGCHI conference in Human factors in computing systems, USA, 2007. 61-70.

[5] Lienhart, R., Abstracting Home Video Automatically. In MULTIMEDIA '99: Proc. of the seventh ACM Int. Conf. on Multimedia (Part 2). ACM, USA, 37-40.

[6] Siegel, S., Castellan, N.J., Nonparametric Statistics for the Behavioral Sciences, 1988, New York, NY. McGraw-Hill.

[7] Wang, P. P., et al., and Zhang, Y., Information-theoretic content selection for automated home video editing. In Image Processing, 2007. ICIP 2007. IEEE Int. Conf. on. Vol. 4. 537-540. USA. 